\title{
Assessment of Posture Related Musculoskeletal Risk Levels in Restaurant Chefs using Rapid Entire Body Assessment (REBA)
}

\author{
Bijal Jignesh Karelia ${ }^{1}$, Deepali Rathod ${ }^{2}$, Ajay Kumar ${ }^{3}$ \\ 'B.P.Th, DPO's NETT College of Physiotherapy, Thane, Maharashtra, India. \\ ${ }^{2}$ Assistant Professor, TMVs Lokmanya Medical College of Physiotherapy, Kharghar \\ ${ }^{3}$ Principal, DPO's NETT College of Physiotherapy, Thane.
}

Corresponding Author: Bijal J Karelia

\begin{abstract}
Background: Objective To assess the level of risk in posture associated work related musculoskeletal disorders in restaurant chefs using Rapid Entire Body Assessment [REBA].

Method: 30 subjects were included. Out of 30 subjects, all of them were males. The Mean Age, Weight, Height and working hours were 19.57 years, $52.06 \mathrm{~kg}, 185.28 \mathrm{cms}$ and 8.63 hours respectively. Postures using REBA score sheet were evaluated based on the most difficult work tasks (based on observation and interview), the posture that was sustained for a longer period of time.

Result: Body posture was evaluated using REBA sheet and it can be concluded that the major areas susceptible to painful working postures were of the neck and the wrist positions having the most frequent risk reported. Followed by the upper arm and trunk position having moderate risk and the least frequent area involved were legs and lower arm position.

Conclusion: The study concludes that most of the restaurant chef's fall under moderate risk of musculoskeletal disorders and requires intervention and awareness about postural correction exercises to prevent further musculoskeletal injuries.
\end{abstract}

Keywords: Restaurant chefs, Posture, Rapid Entire Body Assessment, Ergonomics, Musculoskeletal risk.

\section{INTRODUCTION}

Cooking is a part of day-to-day activity for an individual to lead a life. However, as a profession, they prepare a wide range of foods and recipes in routine manner.

A chef is an incharge of the kitchen in restaurants or places where food is served. ${ }^{[1]}$

Chef needs to have knowledge about food preparation, production and processing.

Chefs also have to order food or kitchen supplies and provide cost estimate to the employers. ${ }^{[2]}$

Professional chefs are open to a variety of learning involving college training and then working from the lowest position in the kitchen towards the top.

Training is essential as the job demands working quickly and effectively under pressure and has appropriate motor skills and manual dexterity.

Restaurants are notorious for their discipline as attention to detail in producing high quality food consistently is essential.

Kitchens are hot and uncomfortable and the chefs have to work a variety of hours such as early mornings, late evenings and even on holidays and weekends. ${ }^{[3]}$

The occupation of chefs is a very fast paced and normally has full time hours. 
The working processes in this profession still largely rely on human work and cannot be replaced by automation. ${ }^{[4]}$

Musculoskeletal disorders are described as an injury or dysfunction that commonly involves the supporting structures of the body as well as the nerves, muscles, bones and cartilages. They are collectively caused by repetitive movements or sustained poor and awkward positions. ${ }^{[5]}$

A chef's work is characterized by long standing hours, constant forward leaning, repetitive upper body movements, lifting heavy objects and awkward postures.

These include grasping of cooking utensils, tossing a wok, cutting vegetables etc. all of which require the forceful exertion of the entire body.

Most of these tasks involve static postures and repetitive movements that can lead a chef at a risk of developing musculoskeletal disorders risk. ${ }^{[6]}$

There are literatures which state that the musculoskeletal disorders in male kitchen workers in South India are high and the areas affected are low back, shoulder, fingers, wrists, knee, neck region, elbow/forearm, upper back. ${ }^{[7]}$

\section{Rapid Entire Body Assessment (REBA) \\ REBA (Rapid Entire Body}

Assessment) was developed by Hignett and MCAttamney in 2000.It is used to assess the entire body postural musculoskeletal disorders and risk associated with the job tasks. A single worksheet is used to evaluate the entire body posture, forceful exertion, type of movement, action and repetitive work. There is no advanced degree or expensive equipment required for assessing. The evaluator just needs REBA worksheet and a pen. The worksheet scores for each of the following body region: wrist, forearm, elbows, shoulder, neck, trunk, back, legs and knee. The score is collected and compiled to form a single score that represents the level of musculoskeletal disorder risk.

Rapid entire body assessment (REBA) has been used to highlight the importance of prevention programs and to improve the working conditions that minimize the damage and improve the quality of life of workers and also productivity of small industries in developing countries. ${ }^{[9]}$

REBA is a convenient assessment scale to assess posture of jobs in several occupational settings, including industrial and health care jobs, construction, sawmill tasks, supermarket industries, school workshops, odontological services and for firefighters and emergency medical technicians.

There are literatures which compared three observational methods to assess ergonomical risk levels that are REBA, RULA and OWAS using data from a sample of 301 postures obtained from diverse industrial sectors. Results showed highest intra-rater reliability of REBA among OWAS and RULA.

REBA method appeared to be the only method capable of capturing the very uncomfortable postures that were frequently observed in a tree nursery sector (squatting, sitting on the ground, lumbar flexion greater than 90 and torso twisting). The study concluded better understanding of the differences between many risks assessment and a useful information for practitioner when choosing a method prior to an ergonomic intervention in an industry.

Corrective actions and ergonomic intervention can be recommended. ${ }^{[10]}$

Since REBA was proven to be freely available assessment scale, which allows assessment of the whole body including lower limbs and is less time consuming; this study aims to use REBA as an outcome measure to evaluate the musculoskeletal risk factors in restaurant chefs.

\section{MATERIALS AND METHODS}

- This study was a cross sectional observational study where 30 restaurant chefs were selected using convenient sampling. Inclusion criteria: Subjects willing to participate, all the subjects in this study were males, the age group of 
30-45 years including that they had a work experience of 6-10 years and worked an average 6-10 hours of work per day. Subjects included in the study were all males. Restaurant chefs having any recent musculoskeletal injuries or deformities, having any neurological problems and a BMI $<18$ and $>25$ was excluded from the study.

\section{PROCEDURE:}

A written consent was taken from all the subjects in the language best understood by them. Selection of the subject was done as per the inclusion and exclusion criteria. Purpose of the study and procedure was explained prior to the study. Demographic data was noted down All the subjects were interviewed and evaluated to understand their work/tasks and demands. The standing postures and movements were observed during their several working cycles.
Postures were evaluated based on the most difficult work tasks (based on observation and interview), the posture that was sustained for a longer period of time and posture in standing where highest loading takes place. REBA is quick, easy, valid, and reliable and less time-consuming method to assess ergonomic posture related musculoskeletal disorders risk. After evaluating and observing the posture bilateral evaluation were done.

\section{REBA (RAPID ENTIRE BODY} ASSESSMENT: - The REBA worksheet is divided into two sections: Section A and Section B. Section A which is on the left side covers the neck, trunk and leg. Section $\mathrm{B}$ which is on the right side covers the arm and wrist. Section A was evaluated first followed by Section B.

\section{MATERIALS:}

1. REBA worksheet.

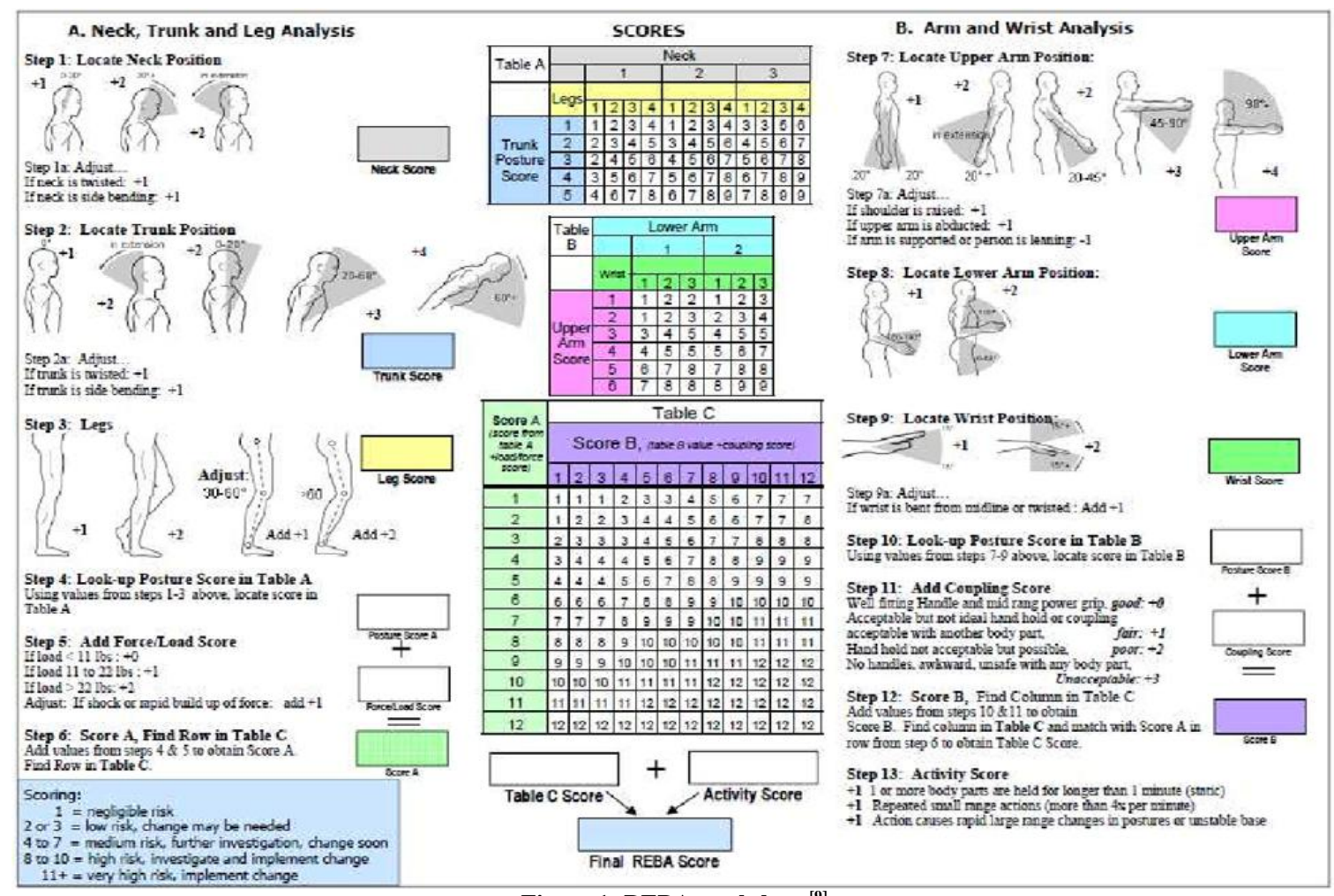

Figure 1: REBA worksheet ${ }^{[9]}$

7. Weighing machine

2. Pen

3. Pencil

4. Scale

5. Adhesive marker

6. Measuring tape

\section{STATISTICAL ANALYSIS:}

Data was collected on a data sheet and encoded for computerized analysis. 
Tables were made using Microsoft word and figures were plotted using Microsoft excel windows 10 .

\section{RESULT}

The highest risk reported area among 30 restaurant chefs was neck $(19.03 \%)$ and wrist position (18.73\%) followed by upper arm position (18.41\%) and trunk position $(17.78 \%)$ and subsequently the leg $(14.60 \%)$ and lower arm position (11.43\%) having the lowest risk.

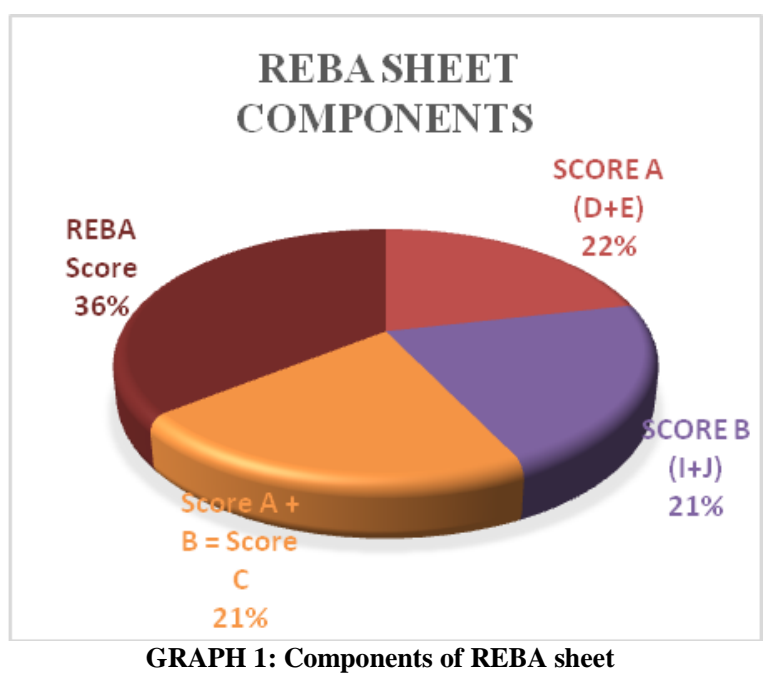

\section{Interpretation:}

- The Above Pie Chart Elucidates that after consolidating the results of REBA score, Score A $(\mathrm{D}+\mathrm{E})$, Score B and Score $\mathrm{C}$ for 30 restaurant chefs, the REBA score has a percentage of $36 \%$, Score A (D+E) has a percentage of $22 \%$, whereas score $\mathrm{B}$ and Score $\mathrm{C}$ have a percentage of $21 \%$ each.

- Score A (D+E) comprises of summed scores for neck, leg and force load.

- Score B comprises of summed scores for Upper arm \& Wrist, and coupling score

- Score C comprises of Score A and Score B.

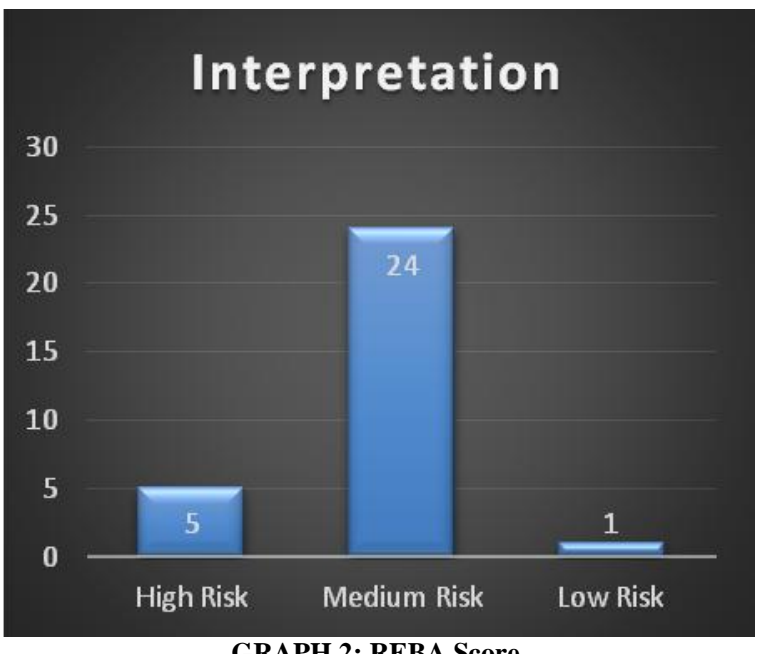

GRAPH 2: REBA Score

TABLE 1: REBA Score

\begin{tabular}{|l|l|l|l|l|}
\hline Reba Score & $\mathbf{1}$ & $\mathbf{2 - 3}$ & $\mathbf{4 - 7}$ & $\mathbf{8 - 1 0}$ \\
\hline Risk Level & Negligible & Low & Medium & High \\
\hline Required action & None & May Be Necessary & Necessary & Necessary soon \\
\hline Percentage of Students & $0 \%$ & $17 \%$ & $80 \%$ & $3 \%$ \\
\hline
\end{tabular}

\section{Interpretation:}

The Above Tabular Data and Bar chart depicts that out of REBA score from $1-10$, out of the 30 restaurant chefs 5 chefs report high risk, 1 chef is at a low risk and the majority 24 out of 30 chefs report medium risk of musculoskeletal disorders.

\section{DISCUSSION}

Position and condition of body or body parts while working is called work posture. Good work posture is important to reduce stress and to increase the efficiency of work. ${ }^{(11)}$
Analyzing the working posture will give the detailed understanding of the workrelated musculoskeletal disorders. ${ }^{(14)}$

Apart from good posture, ergonomic design is also in important for health, safety and efficiency.

This analysis will also assist in early ergonomic intervention if needed.

This study assessed the posturerelated musculoskeletal disorders in the restaurant chefs working in India.

In this study 30 subjects were included. All of them were males. The mean age, weight, height and working hours were 38.23 years, $69.47 \mathrm{kgs}, 167.97 \mathrm{cms}$ and 9.3 
hours respectively. Postures were observed and analyzed in various positions that the chefs find strenuous during cooking using REBA sheet.

Among the musculoskeletal disorders reported the highest risk reported area was neck $(19.03 \%)$ and wrist position $(18.73 \%)$ followed by upper arm position $(18.41 \%)$ and trunk position $(17.78 \%)$ and subsequently the leg (14.60\%) and lower arm position (11.43\%) having the lowest risk.

A study conducted by Subramaniam S. et al on male kitchen workers in the southern region of India using standardized Nordic Musculoskeletal questionnaire. The result of this study reported high risk of work-related musculoskeletal disorders. and the areas affected are low back, shoulder, fingers, wrists, knee, neck region, elbow/forearm, upper back. ${ }^{(7)}$

Activities like lifting heavy kitchen utensils, tossing a wok and prolonged standing made chefs to acquire faulty postures. Also standing and chopping for prolonged period of time has caused them to attain a slouched back posture. Excessive strain is put on their neck. It was found that this constant activity with flexed spine and neck over a period of time might cause a forward head posture in these chefs leading to stretching of the anterior neck muscles and weak neck extensors altering neck biomechanics.

It was identified that the muscles around the shoulders have to work maximum capacity when the upper arm is not supported while stirring food, chopping and even plating which requires fine movements.

No posture or movements ought to be kept at significant stretch of time. The more prominent the muscular effort the shorter the time it is maintained. ${ }^{(12)}$

It was found that almost all the restaurant chefs were at a medium risk if they continue to work in the same posture, which may lead to musculoskeletal disorder risk in future.
Corrective actions and ergonomic intervention are recommended.

The Rapid Entire Body Assessment (REBA) is an ergonomic method which is used for assessing postures.

The application of REBA has evolved over time. It started with photographs, paper and pen. Over time, progress was made using video recordings and employing analysis of this through software. Currently, some equipment is used to measure angles and evaluate in real time.

The advantage of REBA is that it is cost effective and easy to apply with assessment of each body part.

One of the major differences between using REBA is that it assesses the lower extremities as well which is not considered in other evaluation methods like RULA. ${ }^{(13)}$

In a study conducted by David et al REBA was compared with other 14 observational methods to assess ergonomic work-related musculoskeletal disorders risk in terms of main features and functions and exposure factor REBA was concluded to be a freely available assessment scale, which allows assessment of the whole body including lower limbs and the best matched method to the needs of occupational safety and health practitioners who have limited time and resources. ${ }^{(10)}$

REBA method has been applied in 24 different countries and on 91 cases with the highest number of applications in India.

In this study REBA is specifically used to assess an altogether different sector of which involves Restaurant chefs studying in India and their musculoskeletal risk level depending on their working condition and posture. $^{(13)}$

\section{CONCLUSION}

After the interview the standing posture that took the highest load was evaluated using REBA sheet and it can be concluded that the major areas susceptible to uncomfortable and painful working postures were neck and the wrists, having the most frequent risk reported. 
Thus this study concludes that almost all of the restaurant chefs fall under moderate risk of musculoskeletal disorders and require intervention and awarness about postural correction exercises to prevent further musculoskeletal injuries.

\section{Clinical Implication:}

Prevention of injury and musculoskeletal disorders and postural correction exercises should be main concern.

Ergonomic advice and workplace modification like sitting and chopping reducing the load on the neck, physical workspace and environment can improvise work performance.

Also taking frequent breaks in between their work to reduce load on hands should be advised.

\section{AKNOWLEDGEMENTS}

I express my deep sense of gratitude and sincere thanks to our respected sir Dr. Ajay Kumar and guide Dr. Deepali Rathod who immensely helped me with sincere guidance, untiring cooperation, valuable advice and endless inspiration during the course of the study. I also take this opportunity to thank my family members and friends for their help and support.

\section{Conflict of Interest: None}

\section{Source of Funding: None}

\section{Ethical Approval: Approved}

\section{REFERENCES}

1. Subramaniam $\mathrm{S}$, Murugesan $\mathrm{S}$, Jayaraman S. Assessment of shoulder and low back muscle activity of male kitchen workers using surface electromyography. International journal of occupational medicine and environmental health. 2018;31(1):81-90.

2. Crosby PB. Working like a chef. Quality. 1989;28(1):24.
3. Pratten JD. What makes a great chef? British Food Journal. 2003 Aug 1;105(7):454-9.

4. Sant S, Kamthe R, Shaikh $\mathrm{S}$ et al. Prevalence of musculoskeletal pain in cooking professionals of rural versus urban restaurants. Int J Health Sci Res. 2016; 6(11):167-171.

5. Ilyas T, Umer B, Taufiq F. Frequency of Musculoskeletal Disorders and their Related Causative Factors among Female architecture Students in Lahore. Journal of Riphah College of Rehabilitation Sciences. 2018; (1):3842.

6. Shakya NR, Shrestha S. Prevalence of work related musculoskeletal disorders among canteen staff of Kathmandu University. Journal of Kathmandu Medical College. 2018;17(4):162-7.

7. Subramaniam S, Murugesan S. Investigation of work-related musculoskeletal disorders among male kitchen workers in South India. International Journal of Occupational Safety and Ergonomics. 2015 Oct 2;21(4):524-31.

8. Middlesworth MA. Rapid Entire Body Assessment (REBA). Recuperado de: www. ergp-plus. com. 2000.

9. Al Madani D, Dababneh A. Rapid entire body assessment: a literature review. American Journal of Engineering and Applied Sciences. 2016;9(1):107-18.

10. Lascano A, Patín G, Larrea A, San Antonio T. Ergonomic Evaluation of Risk Level by Exposure to Forced Postures in Cattle Slaughterhouse Workers in Ecuador. In International Conference on Applied Human Factors and Ergonomics 2018 Jul 21 (pp. 212217). Springer, Cham.

11. Rahman CM. Study and analysis of work postures of workers working in a ceramic industry through rapid upper limb assessment (RULA). International Journal of Engineering. 2014 Aug;5(3):8269. 
12. Dul J, Weerdmeester B. Ergonomics for beginners: a quick reference guide. CRC press; 2008 May 28

13. Hita-Gutiérrez M, Gómez-Galán M, Díaz-Pérez M, Callejón-Ferre ÁJ. An overview of reba method applications in the world. International Journal of Environmental Research and Public Health. 2020 Jan;17(8):2635.

14. Heeba Zubair Shaikh, Dr. Priyanka Gokhale and Dr Ajay Kumar.2020, Assessment of Posture Related
Musculoskeletal Risk Levels in Architecture Students Using Reba. Int J Recent Sci Res. 11(08), pp. 3942839432.

How to cite this article: Karelia BJ, Rathod D, Kumar A. Assessment of posture related musculoskeletal risk levels in restaurant chefs using rapid entire body assessment (REBA). Int $J$ Health Sci Res. 2021; 11(5): 333-339. DOI: https://doi.org/10.52403/ ijhsr.20210552 